\title{
Retrospective Radiographic Survey of Unconventional Ectopic Impacted Teeth in Al-Madinah Al-Munawwarah, Saudi Arabia
}

\author{
Shadia A. Elsayed ${ }^{1,2 \star}$, Nebras Althagafi ${ }^{3}$, Rayan Bahabri ${ }^{4}$, Mona M. Alshanqiti ${ }^{5}$, Alhanouf M. Alrehaili ${ }^{5}$, Ahmed Salah Alahmadi $^{6}$ \\ ${ }^{1}$ Department of Oral and Maxillofacial Surgery, Dental College and Hospital, Taibah University, Al-Madinah Al-Munawwarah, \\ Saudi Arabia; '2Department of Oral and Maxillofacial Surgery, Faculty of Dental Medicine for Girls, Al-Azhar University, Cairo, \\ Egypt; ${ }^{3}$ Department of Pediatric Dentistry and Orthodontics, College of Dentistry, Taibah University, Medina, Kingdom of \\ Saudi Arabia; ${ }^{4}$ Department of Restorative Dentistry, College of Dentistry, Taibah University, Medina, Kingdom of Saudi Arabia; \\ ${ }^{5}$ Dental College and Hospital, Taibah University, Al-Madinah, Al-Munawwarah, Saudi Arabia; ${ }^{6}$ Prince Muhammad Bin Abdulaziz \\ Hospital, National Guard, Madinah, Kingdom of Saudi Arabia
}

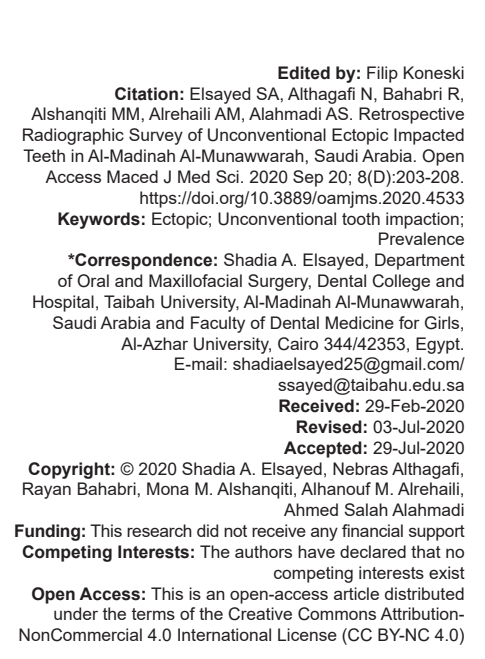

\section{Abstract}

OBJECTIVES: Ectopic unconventional impacted teeth are rare. These teeth erupt in an unusual direction with limited unconventional access and have increased surgical risks.

AIM: This study aimed to investigate and assess the prevalence and distribution of rare ectopic impacted teeth at the Taibah University Dental College and Hospital (TUDCH), Al-Madinah Al-Munawwarah, Saudi Arabia.

METHODS: The study designed through a retrospective radiographic cross-sectional survey involving the review and examination of 9000 archived digital orthopantomograms of patients who visited the (TUDCH) in the period from January 2014 to December 2019 and to analyze any associated factors.

RESULTS: There were 63 ectopically impacted teeth, with an incidence of $0.7 \%$. The age of the patients ranged from 18 to 68 years, with a mean of $32.4 \pm 13$ years. Regarding patient nationality, $68.3 \%$ were Saudis. The most common ectopically impacted teeth were the extra impacted premolars, with an incidence of $0.2 \%$, followed by the inverted molars, impacted first or second molars, and buccoversion or lingoversion third molars, with incidences of $0.16 \%, 0.13 \%$, and $0.12 \%$, respectively. The mandible was affected with ectopic impaction more than the maxilla, with an incidence of $55.6 \%$. There was no difference between the right and left sides. Impacted teeth in the sinus were the least common.

CONCLUSION: The prevalence of ectopic impacted teeth was $0.7 \%$ among the surveyed patients at TUDCH Al-Madinah Al-Munawwarah, Saudi Arabia. Hence, the oral surgeon must have readiness for such a challenging, increasing situation.

\section{Introduction}

Ectopic impacted teeth are rare. They are teeth that remain embedded in an abnormal position, have an unusual eruption pattern, and are not classified as ordinary impaction. They are located in the mucosa, jawbone, maxillary sinus, or nasal cavity for over 2 years after the physiological eruption time and need a special treatment plan for surgical removal [1], [2], [3], [4], [5].

Any tooth can erupt ectopically. The prevalence of ectopic impaction is different for individual teeth and differs across regions [3]. The etiologic factors of ectopic impaction could be due to disturbances in the growth pattern of the individual concerning aging and eruption time because of the presence of genetic components [6] and environmental factors of developmental disturbances [7]. Moreover, tooth impaction is caused by an abnormal lack of space due to small arches, obstructions in the eruption path, bony lesions, or tooth germ malposition due to intrusive trauma. It may be associated with pathological syndromes such as cleidocranial dysplasia, Gardner's syndrome, Gorlin syndrome, or Yunis-Varon syndrome [8].

Ectopic impactions have been reported by many authors [7] as a case report without specific prevalence rates for most involved teeth. The prevalence of ectopic impactions in permanent first molars is $0.2-3.0 \%$, and it was even more infrequent in permanent first premolars. Reported cases of ectopic impactions involved inverted molars [6], deeply impacted tooth in the mandibular ramus [9], and transmigrated teeth near the nasal cavity [10], which are high-risk factors and require specialized access and management.

Appropriate treatment planning involves an accurate diagnosis, thorough clinical and radiographic examinations, and localization of the ectopic impacted tooth concerning its relation to the adjacent 
structures [11], [12]. The management is usually challenging. In cases of no signs or symptoms, followups have been suggested by some conservative researchers. Meanwhile, in pathological cases, radiography for cyst formation, interceptive treatment, surgical extraction, or orthodontic traction can be performed [13], [14], [15].

Although the epidemiology and classification of the most commonly impacted wisdom are thoroughly investigated, also methods and guidelines of its surgical removal are well established; however, the prevalence of other ectopic impacted teeth such as second and first molars still need determination. Recently, we have seen increased cases of ectopic challenging impacted teeth in our institute. The present study aimed to assess the prevalence and distribution of ectopic challenging impacted teeth in patients who visited Taibah University Dental College and Hospital (TUDCH), Saudi Arabia, through a radiographic retrospective survey. This is the first study in Al-Madinah Al-Munawwarah on factors associated with these exotic ectopic impactions.

\section{Material and Methods}

\section{Study design and setting}

This was a retrospective cross-sectional study involving the review and examination of the archived digital orthopantomograms (OPGs) or cone-beam computed tomography (CBCT) of patients who visited the Faculty of Dentistry, Taibah University, Al-Madinah Al-Munawwarah, Saudi Arabia, in the period from January 2014 to December 2019.

\section{Inclusion and exclusion criteria}

Inclusion criteria

We included all clear panoramic X-rays records or CBCT of Saudi and non-Saudi men and women aged 16-60 years who were screened for general examination at the College of Dentistry Clinics and Hospital, Taibah University, for the past 6 years to determine the prevalence of ectopic impaction. All patients who had complete data files were included in the present study.

\section{Exclusion criteria}

We excluded patients who were younger than 16 years or older than 60 years, patients who did not visit the Faculty of Dentistry, Taibah University, in the period from January 2014 to December 2019. Any radiograph with blurred images due to positioning errors was also excluded from the study.

\section{Sampling (type, size, and frame)}

In the absence of ectopic impaction in the region, the sample type used in the present study was a convenient, non-random sample. Two separate investigators examined a total of approximately 9000 available radiographs to identify the presence of ectopic impacted teeth. Patients who had both sets of OPG and CBCT archived on our system were examined and counted once.

\section{Technique and data collection}

Data were collected using the Carestream Clinical and Practice Software (CSR4) system at both female and male sectors. All the patients were radiographed using the same machine (Carestream Health Inc. CS9000Y/3D System (SM764-ES) ED 01/NY, USA). The file number, age, sex, and ethnicity of all included participants were recorded in an excel sheet. Statistical Package for the Social Sciences software (version 16) was used to calculate the prevalence. Ectopic teeth were identified, and their count, site, distribution, relation to the adjacent structures, and the best possible management technique were discussed.

Descriptive statistics were performed, and associated factors were analyzed with inferential statistical tests.

\section{Ethical concerns}

This study was presented to the Ethics Committee of TUDCH for ethical clearance. This was an observational radiographic survey with no human interventions. The need for informed consent was waived, as the identity of the patient was not revealed. (TUCDREC/20181115/Alshanqiti).

\section{Results}

The total study sample included 9000 radiographs and 420 of them were CBCTs, which showed 63 ectopic impacted teeth $(0.7 \%)$. The age of the patients with ectopic impactions ranged from 18 to 68 years, with a mean of $32.4 \pm 13$ years. Regarding patient nationality, $68.3 \%$ were Saudis. The most frequently ectopically erupted teeth were the extra impacted premolars (Figure 1), at $28.6 \%$ and prevalence of 0.2 . The mandible was affected by ectopic impaction more commonly than the maxilla, at $55.6 \%$ (Table 1). 


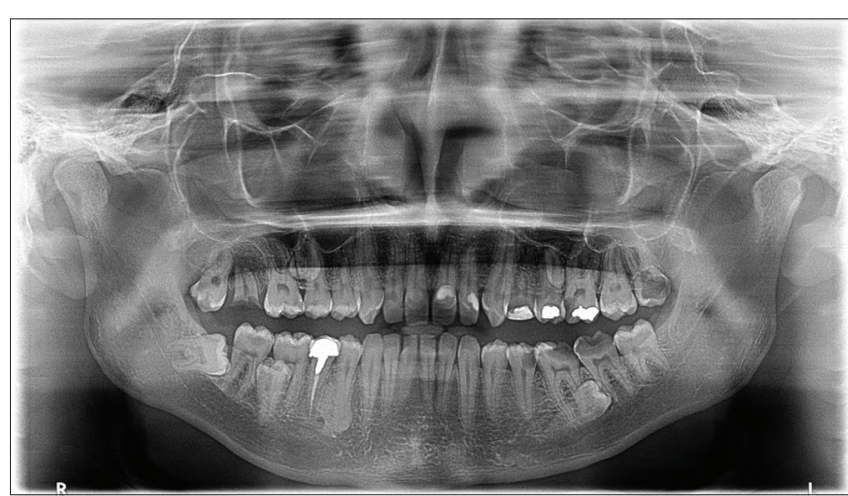

Figure 1: Orthopantomogram (OPG) photograph showing extra impacted mandibular premolars at the right and left side of the OPG

Table 1: Descriptive data of frequency, percentage, and prevalence of various ectopic impacted teeth

\begin{tabular}{lllll}
\hline Variable & Category & Frequency & Percent & Prevalence \\
\hline Ectopic teeth & Extra impacted premolars & 18 & 28.6 & 0.2 \\
& Bucco/lingoversion wisdoms & 11 & 17.5 & 0.12 \\
& Inverted wisdoms & 14 & 22.2 & 0.16 \\
& The tooth in the sinus & 1 & 1.6 & 0.01 \\
& Impacted 6 and 7 & 12 & 19.0 & 0.13 \\
& Rudimentary wisdom & 6 & 9.5 & 0.07 \\
& Edentulous ridges impacted teeth & 1 & 1.6 & 0.01 \\
& Total & 63 & 100.0 & 0.7 \\
\hline
\end{tabular}

There were no differences in ectopic impactions between the right and left sides. The most common ectopic impactions were associated with the extra impacted premolars, especially mandibular, followed by the inverted third molars (Figure 2), impacted first and second molars (Figure 3), and buccoversion of the third molars (Figures 4 and 5 ) at $22.2 \%, 19 \%$, and $17.5 \%$, respectively, and prevalence of $0.16,0.13$, and 0.12 , respectively. Ectopic teeth found in the sinus and impacted teeth in edentulous patients were less common, with a prevalence of 0.01 .

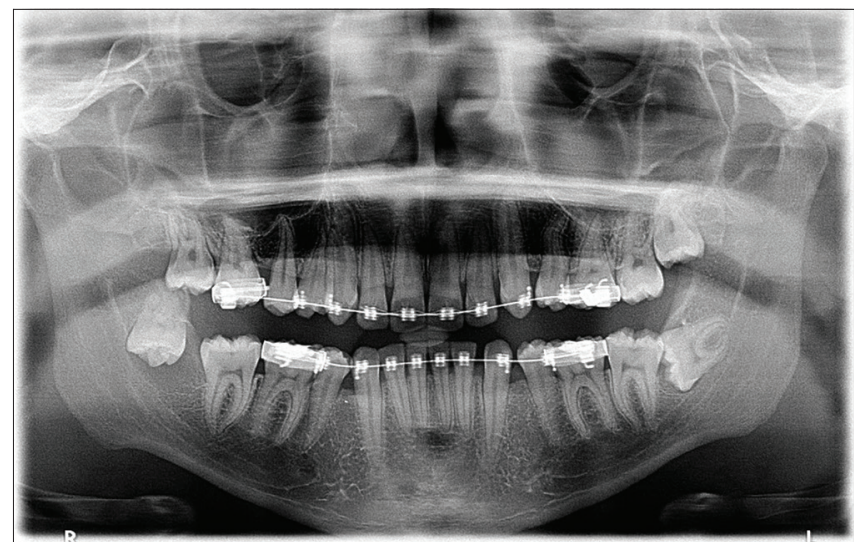

Figure 2: Orthopantomogram photograph showing inverted third molar at the right mandibular ramus
The statistical analysis revealed no significant association between the presence of ectopic impacted teeth and other factor, such as age, sex, site, side, or nationality (Table 2 ).

Table 2: Descriptive data of frequency, percentage, and prevalence of ectopic impacted teeth and relation with other study variables

\begin{tabular}{llllll}
\hline Variable & Category & Frequency & Percent & Prevalence & p-value \\
\hline Gender & Female & 8 & 12.7 & 0.09 & 0.06 \\
& Male & 55 & 87.3 & 0.61 & \\
\multirow{5}{*}{ Nationality } & Total & 63 & 100.0 & 0.7 & \\
& Saudi & 43 & 68.3 & 0.48 & 0.11 \\
& Non-Saudi & 20 & 31.7 & 0.2 & \\
\multirow{5}{*}{ Site } & Total & 63 & 100.0 & 0.7 & 0.10 \\
& Lower & 35 & 55.6 & 0.39 & \\
& Upper & 28 & 44.4 & 0.31 & \\
& Total & 63 & 100.0 & 0.7 & \\
& Right & 29 & 46 & 0.32 & \\
& Left & 32 & 50.8 & 0.36 & \\
& Bilateral & 2 & 3.2 & 0.02 & \\
& Total & 63 & 100 & 0.7 & \\
\hline
\end{tabular}

\section{Discussion}

The present study aimed to investigate and assess the prevalence and distribution of ectopic impacted teeth at TUDCH, Saudi Arabia, through a radiographic retrospective survey. This was the first study in Al-Madinah Al-Munawwarah, so we did not find prevalence rates in this region. The overall incidence in our sample was $0.7 \%$, which is comparable with studies in other countries such as India [7], Rome [16], Sweden [17], and Riyadh [18]. However, this prevalence is much higher than the $0.2 \%$ overall impaction prevalence reported by Alami et al., 2019 [19].

Chapman (1923) was the first to report cases of ectopic eruptions with possible causes such as small arches or deviations in the eruption path of the permanent molars [20]. Transmigration of permanent teeth, especially the mandibular canines, has an incidence of $0.140-0.315 \%$ [9]. Impaction of inverted molars, especially the third molars, is rare [2], [16], [20]. The incidence of impacted inverted molars was $0.16 \%$ in our sample, and the prevalence of impacted first and second molars in previous studies was $0.0-0.06 \%$ [21] and reaching $0.16 \%$ in south India population [16], [17]; however, they represented $0.13 \%$ of the sample in the present study, which was comparable to other regions. Other studies reported an increase prevalence rate
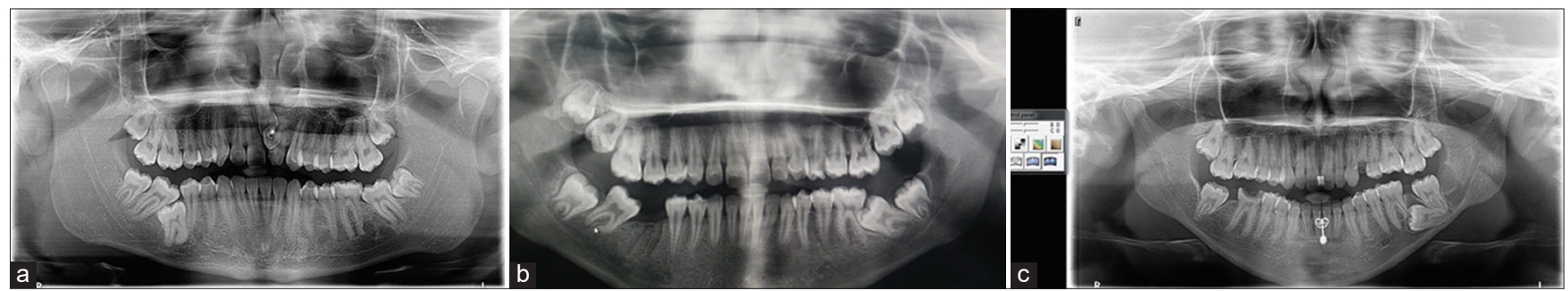

Figure 3: (a) Orthopantomogram photographs showing (a) impacted the first molar at the right side of the mandible, while (b and c) showing impacted second molars at the left mandibular side 
to reach $1.3 \%$ [16]. Therefore, more cases of ectopic impaction are expected, which would need specialized surgical intervention.

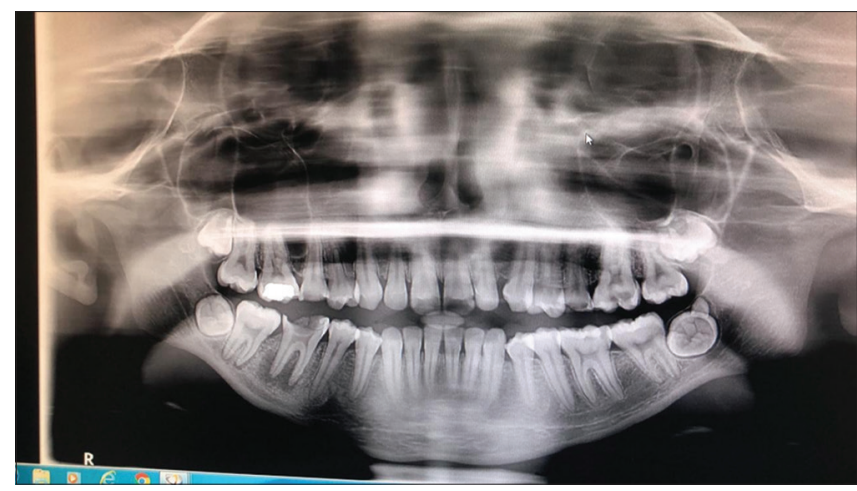

Figure 4: Orthopantomogram photograph showing bilateral buccoversion mandibular impacted wisdom

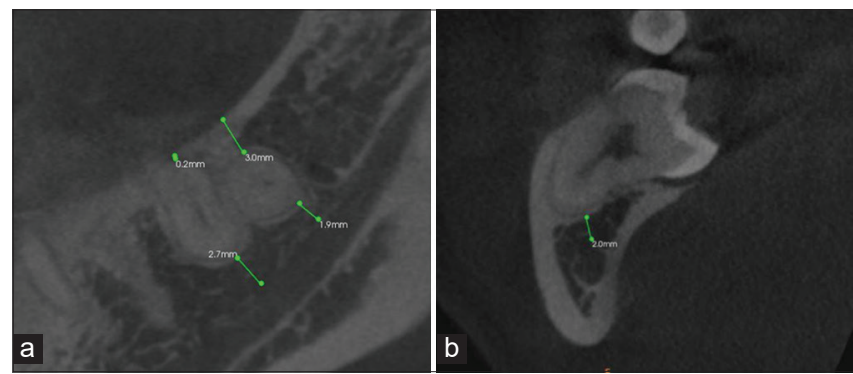

Figure 5: ( $a$ and b) Cone-beam computed tomography photograph showing lingoversion impacted wisdom at the left side of the mandible

Impaction commonly involves the mandibular third molars, followed by the maxillary third molars, permanent maxillary canines, and mandibular premolars. There are different theories on the cause of impaction. Ectopic impactions can cause mild complications such as hyperplastic dental follicles, dentigerous cysts, and odontogenic keratocysts, and severe complications such as squamous cell carcinomas and mucoepidermoid carcinomas [3], [22], [23].

Edentulous jaws had a very rare prevalence rate of impaction in the searched literature [24] and in our current study population, only $0.01 \%$ had impacted teeth.

In a case report from India (2015), a 26-yearold man with multiple missing teeth, which were extracted because of caries and periodontal disease, showed the presence of a tooth in the longitudinal direction in the mid-ramus region of the left jaw on a panoramic radiograph. The patient was asymptomatic and unaware of the tooth [9].

Another study from India (2012) reported four cases of inverted impacted teeth, involving the two maxillary third molars and two inverted supernumerary teeth in the anterior maxilla, and the patients were asymptomatic [6]. This was following our results, which showed that most ectopic impactions are discovered incidentally and are asymptomatic.

Kumar et al., in 2012, [25] reported a case of bilateral transmigrated impacted maxillary lateral incisors and canines. CT revealed that all teeth were impacted horizontally and had migrated across the midpalatine suture, with their cusp tips impacting the root apex of the left central incisor, which was associated with a peri-coronal radiolucency, suggestive of an odontogenic cyst.

Ectopically migrated impaction is rare worldwide and is mostly detected incidentally. For management, some conservative dentists advise the patient to leave the teeth in place, with no interventions, because of lack of pain, discomfort, and the teeth being completely embedded in the bone. However, some surgeons consider risks of pathological fracture and development of pathosis and suggest surgical extraction, especially in symptomatic patients, despite the challenge. In some cases, extraoral approaches, endoscopic assistance, staged orthodontic surgical extraction, and combined intraoral and extraoral approaches [11], [12] or the use of sagittal split osteotomies [26] may be suitable.

Panoramic radiography is the standard method for the preoperative diagnosis of impaction; furthermore, cone-beam CT can be used to overcome the limitations of conventional radiography [27]. However, successful treatment of impacted teeth requires interdisciplinary management, involving general dentists and surgery specialists [28]. Some dentists prefer coronectomy to complete the extraction of complicated impacted teeth. The principal indication of coronectomy is to avoid iatrogenic inferior alveolar nerve injuries when extracting third molars [2], [16], [20], [29]. The contraindications of coronectomy include necrotic third molars, extensive caries with the risk of pulpal involvement, tooth mobility, periapical disease, and presence of cystic lesions, which are unlikely to resolve because of retention of the tooth inside the bone, and tumors. According to Gady and Fletcher, horizontal impaction and pericoronal or periapical infections are the contraindications for coronectomy [29]. Other contraindications may include immunocompromised patients, a history of radiotherapy in the head and neck or treatment before radiotherapy, neuromuscular disorders, diabetes mellitus, and osteosclerosis/osteopetrosis of the jaws. According to Renton et al. [30], the use of immunomodulating drugs is a contraindication for coronectomy, as patients on long-term prednisolone have a high risk of ulcerative colitis.

Maxillofacial surgeons, orthodontists, and general dentists may refer to this study to review the prevalence of different impaction types. Ectopic challenging impacted teeth had increased recently in the reported case reports. The precise identification of the most likely teeth to be affected, the shape and location of the teeth, and relationship of the ectopically impacted teeth with the adjacent structures are important factors in the success of the removal process and understanding treatment options, as each ectopically impacted tooth requires a unique surgical approach for removal to avoid postoperative 
complications. Therefore, the dental surgeon must examine the $\mathrm{X}$-rays and acquire sufficient information to facilitate the process of removal.

\section{Conclusion}

The prevalence of ectopic impacted teeth was $0.7 \%$ among the patients surveyed at TUDCH. This result would increase the awareness of oral surgeons and dental students about the new exotic impaction era, with undetermined academic classification and patterns unlike the documented well-studied relations of impacted third molars such as positions and angulation (mesioangular, horizontal, such as Pell and Gregory, winter's classification, etc.). Future studies should categorize these ectopic types to determine ideal surgical protocol or guidelines for each degree of difficulty or possibility of complications during the removal process of these ectopic impactions.

\section{Acknowledgments}

The authors would like to express their appreciation to the Engineer. Bilal Khaled Alshantoot and Bashaer Abdul Kareem Alhejaily at TUDCH for their great help in data archive adjustment and collection. Authors would like to express their appreciation to Dr. Tamer Hifnawy, Professor of Public Health and Community Medicine in the College of Dentistry at Taibah University, and Beni-Suef University, Egypt, for his great help and effort in the research course and statistical analysis.

\section{Authors' Contributions}

SAE contributed to conception, design acquisition, analysis, and interpretation of data, drafted, and critically revised the manuscript. RB, $\mathrm{NA}$, and MA contributed to the acquisition, analysis, and interpretation of data, AMA and ASA drafted the manuscript and collected the data. All authors agree to be accountable for all aspects, integrity of the work, and gave final approval.

\section{Ethics approval}

This study was presented to the Ethics Committee of TUDCH for ethical clearance, as this was an observational radiographic survey with no human interventions. The need for informed consent was waived, as the identity of the patient was not revealed. (TUCDREC/20181115/Alshanqiti).

\section{Availability of data and materials}

Data are available on request.

\section{References}

1. Kumar VR, Yadav P, Kahsu E, Girkar F, Chakraborty R. Prevalence and pattern of mandibular third molar impaction in eritrean population: A retrospective study. J Contemp Dent Pract. 2017;18(2):100-6. https://doi.org/10.5005/ jp-journals-10024-1998

PMid:28174361

2. Patil S, Doni B, Kaswan S, Rahman F. Prevalence of dental anomalies in indian population. J Clin Exp Dent. 2013;5(4):1836. https://doi.org/10.4317/jced.51119

PMid:24455078

3. Wang CC, Kok SH, Hou LT, Yang PJ, Lee JJ, Cheng SJ, et al. Ectopic mandibular third molar in the ramus region: Report of a case and literature review. Oral Surg Oral Med Oral Pathol Oral Radiol Endod. 2008;105(2):155-61. https://doi.org/10.1016/j. tripleo.2007.04.009

PMid: 17764987

4. Singh YK, Adamo AK, Parikh N, Buchbinder D. Transcervical removal of an impacted third molar: An uncommon indication. J Oral Maxillofac Surg. 2014;72(3):470-3. https://doi. org/10.1016/j.joms.2013.09.031

PMid:24246255

5. Msagati F, Simon EN, Owibingire S. Pattern of occurrence and treatment of impacted teeth at the Muhimbili National Hospital, Dar es Salaam, Tanzania. BMC Oral Health. 2013;13:37. https:// doi.org/10.1186/1472-6831-13-37

PMid:23914842

6. Mohan S, Kankariya H, Fauzdar S. Impacted inverted teeth with their possible treatment protocols. J Maxillofac Oral Surg. 2012;11(4):455-7. https://doi.org/10.1007/s12663-011-0307-9 PMid:24293940

7. Yaseen S, Naik S, Uloopi K. Ectopic eruption-a review and case report. Contemp Clin Dent. 2011;2(1):3-7. https://doi. org/10.4103/0976-237x.79289

PMid:22114445

8. Kjær I. Mechanism of human tooth eruption: Review article including a new theory for future studies on the eruption process. Scientifica (Cairo). 2014;2014:341905.https://doi. org/10.1155/2014/341905

9. Tarsariya VM, Jayam C, Parmar YS, Bandlapalli A. Unusua intrabony transmigration of mandibular canine: Case series (report of 4 Cases). BMJ Case Rep. 2015;2015:bcr2014205398. https://doi.org/10.1136/bcr-2014-205398 PMid:26361803

10. Kim M, Huh KH, Yi WJ, Heo MS, Lee SS, Choi SC. Evaluation of accuracy of $3 \mathrm{D}$ reconstruction images using multi-detector CT and cone-beam CT. Imaging Sci Dent. 2012;42(1):25-33. https://doi.org/10.5624/isd.2012.42.1.25

PMid:22474645 
11. Wassouf A, Eyrich G, Lebeda R, Gratz KW. Surgical removal of a dislocated lower third molar from the condyle region: Case report. Schweiz Monatsschr Zahnmed. 2003;113(4):416-20. PMid: 12768887

12. Procacci P, Albanese M, Sancassani G, Turra M, Morandini B, Bertossi D. Ectopic mandibular third molar: Report of two cases by intraoral and extraoral access. Minerva Stomatol. 2011;60(7-8):383-90.

PMid:21709653

13. Elsayed S, Alolayan A, Farghal L, Ayed Y. Generalised hypercementosis in a young female seeking extraction: Revision and update of surgical technique. J Coll Physicians Surg Pak. 2019;29(11):1111-3. https://doi.org/10.29271/ jcpsp.2019.11.1111 PMid:31659974

14. Elsayed SA, Gawish AS, Khalifa A. Management of periodontal defect after mandibular third molar extraction. Int $\mathrm{J}$ Clin Oral Maxillofac Surg. 2015;1(1):4-10.

15. Elsayed SA, Ayed Y, Alolayan AB, Farghal LM, Kassim S. Radiographic evaluation and determination of hypercementosis patterns in Al-Madinah Al-Munawwarah, Saudi Arabia: A retrospective cross-sectional study. Niger J Clin Pract. 2019;22(7):957-60. https://doi.org/10.4103/njcp.njcp_614_18 PMid:31293261

16. Cassetta M, Altieri F, di Mambro A, Galluccio G, Barbato E. Impaction of permanent mandibular second molar:Aretrospective study. Med Oral Patol Oral Cir Bucal. 2013;18(4):564-8. https:// doi.org/10.4317/medoral.18869

PMid:23524438

17. Bondemark L, Tsiopa J. Prevalence of ectopic eruption, impaction, retention and agenesis of the permanent second molar. Angle Orthod. 2007;77(5):773-8. https://doi. org/10.2319/072506-306.1 PMid:17685771

18. Afify AR, Zawawi $\mathrm{KH}$. The prevalence of dental anomalies in the Western Region of Saudi Arabia. ISRN Dent. 2012;2012:837270. https://doi.org/10.5402/2012/837270 PMid:22778974

19. Alami S, Aghoutan H, Bellamine M, Quars FE. Impacted first and second permanent molars: Overview. In: Human TeethKey Skills and Clinical Illustrations. London: Intech Open; 2019. https://doi.org/10.5772/intechopen.86671

20. Hafiz ZZ. Ectopic eruption of the maxillary first permanent molar: A review and case report. J Dent Heal Oral Disord Ther. 2018;9(2):154-8.

21. Palma C, Coelho A, González Y, Cahuana-Cárdenas A. Failure of eruption of first and second permanent molars. J
Clin Pediatr Dent. 2003;1:239-45. https://doi.org/10.17796/ jcpd.27.3.dm4v13441p161928

22. Kupferman SB, Schwartz HC. Malposed teeth in the pterygomandibular space: Report of 2 Cases. J Oral Maxillofac Surg. 2008;66(1):167-9. https://doi.org/10.1016/j. joms.2006.09.005

PMid: 18083435

23. Lambade P, Lambade D, Dolas RS, Virani N. Ectopic mandibular third molar leading to osteomyelitis of condyle: A case report with literature review. Oral Maxillofac Surg. 2013;17(2):127-30. https://doi.org/10.1007/s10006-012-0346-5 PMid:22847038

24. Ehara H, Yamaguchi H, Aoki K. Cases of impacted teeth in edentulous patients (4). 3 cases of impacted maxillary premolars. Shikwa Gakuho. 1984;84(4):667-71. PMid:6591437

25. Kumar S, Urala AS, Kamath AT, Jayaswal P, Valiathan A Unusual intraosseous transmigration of impacted tooth. Imaging Sci Dent. 2012;42(1):47-54. https://doi.org/10.5624/ isd.2012.42.1.47

PMid:22474648

26. Catherine Z, Scolozzi P. Mandibular sagittal split osteotomy for removal of impacted mandibular teeth: Indications, surgical pitfalls, and final outcome. J Oral Maxillofac Surg. 2017;75(5):915-23. https://doi.org/10.1016/j.joms.2016.12.039 PMid:28142008

27. Alqerban A, Jacobs R, Fieuws S, Willems G. Comparison of two cone beam computed tomographic systems versus panoramic imaging for localization of impacted maxillary canines and detection of root resorption. Eur J Orthod. 2011;33(1):93-102. https://doi.org/10.1093/ejo/cjq034

PMid:21270321

28. Juvvadi S, Medapati Rama HR, Anche S, Manne R, Gandikota C. Impacted canines: Etiology, diagnosis, and orthodontic management. J Pharm Bioallied Sci. 2012;4(2):S234-8. https:// doi.org/10.4103/0975-7406.100216 PMid:23066259

29. Gady J, Fletcher MC. Coronectomy: Indications, outcomes, and description of technique. Atlas Oral Maxillofac Surg Clin North Am. 2013;21(2):221-6.

PMid:23981497

30. Renton T, Hankins M, Sproate C, McGurk M. A randomised controlled clinical trial to compare the incidence of injury to the inferior alveolar nerve as a result of coronectomy and removal of mandibular third molars. Br J Oral Maxillofac Surg 2005;43(1):7-12. https://doi.org/10.1016/j.bjoms.2004.09.002 PMid:15620767 